\title{
"Siempre nos miran a los del fondo". Zonificación del espacio y producción de identidades en la escuela secundaria
}

'They Always Look to the Back Row'. Zoning of Space and Identity Production in Secondary School

"Eles sempre olham para os que estão no fundo." Zoneamento espacial e produção de identidade no ensino médio

Guido García-Bastán* (iD) hetps://orcid.org/0000-0001-5324-5931

Marina Edith Tomasini** (iD) hteps://orcid.org/0000-0003-4357-287X

Doi: 10.17227/rce.num78-8383

Para citar este artículo: García-Bastán, G. y Tomasini, M. E. (2020). "Siempre nos miran a los del fondo". Zonificación del espacio y producción de identidades en la escuela secundaria. Revista Colombiana de Educación, 78, 15-41. http://doi.org/10.17227/rce.num78-8383

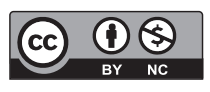

Recibido: 05/09/2018

* Doctor en Psicología. Becario Posdoctoral del Instituto de Investigaciones Psicológicas (IIPSi Conicet, UNC). Correo electrónico: guidogarciabastan@unc.edu.ar

** Doctora en Psicología. Investigadora Adjunta del Consejo Nacional de Investigaciones Científicas y Técnicas - Instituto de Humanidades (IDH - Conicet) Córdoba, Argentina. Correo electrónico: marinatomasini@hotmail.com 


\section{Resumen}

En este artículo de investigación analizaremos la segregación hacia un grupo de jóvenes en el interior de un curso escolar en Córdoba (Argentina), a quienes sus compañeros nombraban despectivamente mediante el modismo local de brasas. Se estructura en dos partes: en la primera, explicitamos las principales referencias teóricas y consecuentes decisiones metodológicas empleadas para abordar los procesos de socialización, sociabilidad y construcción de identidades juveniles en la escuela desde una perspectiva cultural y dialógica. En la segunda, presentamos los principales hallazgos de la investigación. Comenzaremos aproximando al lector al fenómeno de la zonificación del espacio escolar para llegar a plantear dos cuestiones nodales: por un lado, la relevancia de dicha zonificación como artefacto identitario, y por otro, cómo en nuestro caso de estudio esta dimensión se yuxtapuso a otros recursos culturales, contribuyendo a la estabilización de la identidad devaluada del grupo estudiado, pero también dando márgenes a la emergencia de agenciamientos singulares en sus posicionamientos identitarios. Nuestros hallazgos abonan la necesidad de ampliar la mirada investigativa para entender el espacio escolar en su imbricación con otros entornos y mundos figurados.

\section{Palabras clave}

jóvenes; socialización; sociabilidad; escuelas secundarias; zonificación; Argentina

\section{Keywords}

youngsters; socialization; sociability; secondary schools; zoning; Argentina

\begin{abstract}
In this research paper, we analyze the segregation of a group of youngsters within a school year in Córdoba, Argentina, whom their classmates disparagingly named "brasas", an adjective from the local slang. The article is structured in two parts. In the first one, we explain the main theoretical references and consequent methodological decisions used to address the processes of socialization, sociability and construction of youth identities at school from a cultural and dialogic perspective. In the second part, we present our main findings. We will begin by approaching the reader to the phenomenon of the zoning of school space to reach two nodal statements. On the one hand, the relevance of such zoning as an identity device. On the other hand, how in our case study this dimension was combined with other cultural resources, contributing to stabilize the devalued identity of this group, but also allowing agency in their identity positions. Our findings suggest the need to broaden the investigative perspective to understand the school space in its imbrication with other spaces and figured worlds.
\end{abstract}

\section{Palavras-chave}

juventude; socialização; sociabilidade; escolas secundárias; zoneamento Argentina 


\section{Introducción}

Los entornos espaciales no son un mero escenario o telón de fondo de la vida social: el espacio y las prácticas se producen mutuamente (Giddens, 1984; Lefebvre, 1991). Sabemos que los posicionamientos espaciales (entre otras prácticas de posicionamiento) tienen consecuencias sobre la producción de identidades (Holland y Leander, 2004) y que la organización del espacio y su convencionalización fueron piezas basales de nuestros sistemas educativos (Álvarez y Varela, 1991; Puiggrós, 1990). Sin embargo, las etnografías escolares aún no han abordado de modo decidido las prácticas de posicionamiento en el espacio y sus relaciones con la producción identitaria (Leander, Phillips y Headrick, 2010).

Históricamente, la gestión del espacio ha sido una de las claves del control disciplinario en el ámbito escolar. No referimos solo a la arquitectura panóptica (Foucault, 2008) o a la distinción categórica entre áreas y momentos de trabajo y de ocio, sino también a la delimitación de posiciones en una geografía imaginada del aula (Leander, Phillips y Headrick, 2010). Las genealogías escolares sugieren que los estudiantes del fondo y de la primera fila han existido siempre. El dinámico sistema lancasteriano, por ejemplo, distinguía de este modo a quienes se encontraban rezagados o avanzados en la carrera por el aprendizaje, mientras que jesuitas y lasallanos dirimían la ubicación de cada estudiante en función de los méritos que mostrase, ejerciendo prácticas de vigilancia (Dussell y Caruso, 1999; Narodowski, 2008).

En la literatura anglosajona es posible reconocer, con especial vigor a partir de la década de 1980, un interés por el aula en los estudios del classroom management: un área estructurada en torno a la optimización de un ambiente propicio para los aprendizajes y el engagement con las tareas escolares (Barrett, Davies, Zhang y Barrett, 2015; Evertson y Weinstein, 2011; Nash, 1981; Rosenfield, Lambert y Black, 1985; Weinstein, 1981). Se trata, generalmente, de estudios que reportan mediciones en las que la clase y los aprendizajes son tratados como fenómenos aislados de su contexto cultural.

También la dimensión espacial ha sido atendida, generalmente de modo tangencial o como tema secundario, por estudios interesados en procesos psicosociales y culturales enlazados a la escuela, que ponen atención a su zonificación en relación con prácticas sociales rutinizadas (Giddens, 1984). Por ejemplo, documentando la persistencia de una zonificación del aula que demarca regiones de control o castigo (Rist, 1970; Saucedo, 2005), mostrando cómo la masculinidad y feminidad hegemónicas configuran usos juveniles diferenciales de los espacios escolares y sus regiones (Beer, 2008; Wenetz y Stigger, 2011) o señalando que la disposición espacial al interior 
de las aulas puede revelar fronteras intergrupales juveniles, a veces ligadas a taxonomías sociales racializadas que intervienen en la producción identitaria (Leander, 2002; Maldonado, 2006).

En este escrito recuperamos el interrogante referido a las relaciones entre las dimensiones espacial e identitaria en el contexto escolar, con el objetivo de aportar algunas respuestas que contribuyan a complejizar la comprensión de la imbricación entre ambas. Si bien no nos centraremos en los procesos de aprendizaje, nuestros análisis pueden significar una contribución a ciertas perspectivas ecológicas (Brito Rivera, Subero y Esteban-Guitart, 2018; Esteban-Guitart y Moll, 2014) que ven en la indagación de los procesos de construcción de identidad una clave para el análisis de los procesos educativos, ya que permite establecer continuidades entre diversas experiencias de aprendizaje tanto dentro como fuera de la escuela.

Comenzaremos por enfocarnos en procesos que ocurren en el interior del aula, para llegar a mostrar lo injusto del recorte. Nuestra apuesta intentará, además, integrar los dominios que en algunas de las investigaciones citadas aparecen abordados de modo algo inconexo; por un lado, la zonificación del aula como resultado de prácticas juveniles de sociabilidad y, por otro, su empleo en la regulación de la disciplina y los aprendizajes por parte de los educadores.

A partir de un estudio de caso en la ciudad de Córdoba (Argentina), analizaremos el proceso de segregación hacia cinco jóvenes de un curso escolar. Situación de la que llegamos a tener conocimiento siguiendo la pista de las significaciones que el territorio de aula tenía para docentes y estudiantes. Dado que la estigmatización entre estudiantes es una forma de violencia simbólica que ha sido relacionada ya con la construcción de identidades juveniles (Gómez Cabrera, 2016), nos interesa mostrar, por un lado, la relevancia que puede cobrar la zonificación del aula como artefacto identitario (Leander, 2002) y, por otro, cómo en nuestro caso de estudio esta dimensión se yuxtapuso a otros recursos culturales (escolares y no escolares) contribuyendo a la estabilización de ciertas identidades devaluadas, pero también dando margen a la emergencia de lo que interpretamos como agenciamientos singulares en la constitución identitaria o prácticas de subjetivación. Fundamentar esta proposición implicará un esfuerzo por atender paralelamente a los procesos de socialización escolar y sociabilidad juvenil, argumentando que ambos intervienen activa y conjuntamente en la producción de identidades socioescolares. Subsidiariamente, nuestros hallazgos abonan una línea interpretativa que alienta a pensar a la escuela en un nexo de relaciones espaciales que exceden lo que comúnmente se recorta como parte del ámbito escolar (Leander, Phillips y Headrick, 2010). 


\section{Socialización, sociabilidad y producción de identidades en mundos figurados}

En sentido amplio, la socialización y la sociabilidad aluden a modos de aproximarnos a referencias culturales y simbólicas en la constitución de nuestra identidad personal y social. La sociología del conocimiento de Berger y Luckmann (2001) veía en la socialización un proceso por el cual el individuo se volvía miembro de una sociedad constituyendo su identidad (self). Un rasgo que distinguió a esta formulación de sus contemporáneas funcionalistas, fue el énfasis en lo inacabado e indeterminado del proceso: a lo largo de nuestra vida incorporamos y recreamos un conocimiento de normas, valores y roles que, en sociedades diferenciadas, se correspondería con submundos institucionales cada vez más especializados. Pervive en esta conocida formulación un sesgo institucionalista, quizá parcialmente responsable de que en la investigación educativa generalmente el estudio de la socialización haya sido equiparado con el estudio del aprendizaje del rol estudiantil, bajo la orientación e iniciativa de los educadores. Aunque en algunas oportunidades, reconociendo que la construcción del oficio de estudiante no se agota en una inducción unilateral por parte de los adultos, el concepto haya sido utilizado para dar cuenta de aprendizajes en que los pares se constituyen como otros significativos (Paulín y Tomasini, 2007; Vásquez y Martínez, 1996).

A veces solapado con esta concepción ampliada de la socialización, el concepto simmeliano de sociabilidad designa relaciones entre sujetos que se visualizan -al menos situacionalmente- como pares. Al referir a la sociabilidad juvenil suelen destacarse sus componentes lúdicos y afectivos por sobre los racionales (Maffesoli, 1990; Weiss, 2009), sin que por ello deje de pensarse como una instancia reflexiva que permita romper o cuestionar las normas del mundo adulto, contribuyendo a la subjetivación (Mejía y Weiss, 2011). Lo que implica reconocer en el actor a un sujeto crítico, irreductible a sus roles e intereses (Dubet, 2010). Otro aspecto señalado como propio de la sociabilidad en los jóvenes es la centralidad que asume el conocimiento del otro diferente (Weiss, 2009). Tarea en la que a veces la violencia cumple un papel importante como herramienta de conocimiento y búsqueda de reconocimiento, constituyéndose así la identidad en las interacciones con los otros (García y Madriaza, 2005; Paulín, 2015; Tomasini, López, Bertarelli y García Bastán, 2010).

En este sentido, el enfoque dramatúrgico de Goffman (1989) consideraba a la identidad, o el sí mismo (self), como producto emergente de las situaciones de interacción. Si el autor consiguió exceptuarse de postular un interior psíquico al referir al self, ello se debe a que su oficio sociológico lo encontraba ocupado en responder a la pregunta por el orden de la interacción (Goffman, 1983) al margen de las motivaciones singulares que 
los actores pudiesen atribuir a sus acciones. Sin embargo, desde un enfoque psicosocial crítico (lbáñez, 1992), nos interesa trascender una mirada sistémica para explorar experiencias singulares de producción identitaria a partir de las interacciones. Una perspectiva cultural y dialógica de las identidades (Holland, Lachicotte, Skinner y Cain, 1998) permite atender a los modos en que diversos recursos culturales se articulan a la experiencia. Las interacciones -tal como apuntaba Goffman- enfrentan a los actores a posiciones de sujeto. Pero no lo hacen por fuera de una trayectoria de posicionamientos previos. Esto implica pensar que cada nuevo episodio de posicionamiento se vincula a sus antecesores, como capas que dan espesor a identidades singulares, contribuyendo a su estabilización (Leander, 2002). Desde la perspectiva que asumimos, dichas experiencias de posicionamiento son momentos cruciales en que los fenómenos sociales y psicológicos se interpenetran, modelando la identidad de un individuo o grupo. Así, nos alejaremos tanto de pensar que la identidad reposa sobre la eficacia de orientaciones normativas preexistentes, como de concebir un actor que responde siempre creativamente desde su posición subjetiva.

Recuperaremos una concepción de las identidades como producidas por y productoras de mundos figurados (Holland et al., 1998). Un término que nos permite ampliar los procesos de inducción y recreación más allá de las fronteras institucionales que preocupaban a Berger y Luckmann (2001). Referimos a esferas de interpretación social y culturalmente construidas en las que personajes y actores particulares son reconocidos, ciertos actos son significados y algunos desenlaces son valorados por sobre otros (por ejemplo, el mundo del romance, el del crimen o el de la academia). En cada mundo figurado, existen agentes que participan de una gama limitada de actos significativos, movidos por un conjunto específico de fuerzas y apoyándose en una serie de artefactos mediadores de sus actividades. En este sentido, la noción de artefactos identitarios (Leander, 2002), subsidiaria de los enfoques socioculturales de la actividad, nos permitirá identificar una serie de instrumentos pertenecientes a estos mundos figurados (simbólicos, materiales y del orden de las prácticas) mediando en la producción identitaria. Esto nos conducirá hacia una interpretación multimodal de las interacciones, atenta a signos lingüísticos, espacios materiales, gestos y objetos en los que los interactuantes se apoyan para condensar y estabilizar significados.

En resumen, entenderemos al self como producto emergente de interacciones sociales. Reconociendo también que, por la mediación de ciertos artefactos culturales, las identidades consiguen estabilizarse de modos diversos. A lo largo de nuestra reconstrucción etnográfica procuraremos atender especialmente a este proceso de estabilización, reconociendo también algunos márgenes de agenciamiento que los actores encuentran para operar sobre sus mundos figurados y subjetivarse. 


\section{Consideraciones metodológicas}

Este trabajo forma parte de los resultados de una tesis doctoral ya concluida (García Bastán, 2017), que abordó algunos aspectos de la conflictividad cotidiana en las interacciones entre docentes y estudiantes de escuela secundaria. Desde un paradigma interpretativo-cualitativo (Vasilachis, 2007) buscamos comprender el sentido de la acción social en el contexto del mundo de la vida y desde la perspectiva de los participantes. En dicha investigación, trabajamos en dos establecimientos educativos de la ciudad de Córdoba (Argentina), de características socialmente contrastantes: una escuela provincial de gestión pública a la que asisten jóvenes de clase baja y una escuela privada de modalidad bilingüe a la que concurren jóvenes de sectores altos de la ciudad. Con un enfoque etnográfico nos aproximamos a dos cursos en cada establecimiento, procurando que en ambos contextos estuviesen representados los dos ciclos que organizan la escuela secundaria argentina: Ciclo Básico $\left(1^{\circ}, 2^{\circ}\right.$ y $3^{\circ}$ año $)$ y Ciclo de Especialización $\left(4^{\circ}, 5^{\circ}\right.$, y $6^{\circ}$ año). En cada una de estas aproximaciones, comenzamos realizando observaciones participantes que nos permitieron familiarizarnos con las rutinas cotidianas e identificar temas en torno a los cuales se suscitaban conflictos. Así, si bien en todos los cursos las entrevistas y conversaciones con docentes y estudiantes indagaron la calidad percibida en los vínculos y los sentidos acerca de la conflictividad, la indagación observacional previa permitió reorientar los temas abordados hacia situaciones concretas que estaban teniendo lugar en cada contexto de observación. En ese sentido, la dimensión espacial de la que aquí nos ocupamos apareció más claramente como tema emergente en el trabajo en uno de los cursos seleccionados.

Los análisis y el material de campo que presentamos aquí refieren al curso de Ciclo Básico de la escuela pública que denominaremos escuela "Güemes". Los jóvenes con los que trabajamos en este artículo pertenecían al turno matutino y se encontraban cursando primer año. Este último dato es metodológicamente relevante: al tratarse de una escuela que solo contaba con el nivel medio, los estudiantes de este curso procedían de distintas escuelas primarias de la ciudad. En la mayor parte de los casos no existían lazos previos entre los jóvenes, lo que nos ubicaba ante un momento de sus trayectorias escolares particularmente propicio para observar la configuración de posicionamientos sociales y producción de identidades.

Los estudiantes de esta escuela proceden de barrios de la zona sur de Córdoba, la región más pobre de la ciudad. Mayoritariamente, son la primera generación de sus familias que cursa estudios secundarios. Como señaló Ziegler (2016), en un escenario argentino educativamente fragmentado, donde existen tipos de escuelas destinadas a grupos sociales con referencias normativas y culturales propias (Tiramonti, 2004), las aulas son internamente

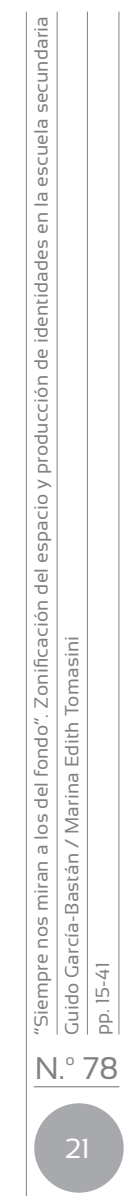


más homogéneas en cuanto a la composición de su alumnado. Al conversar con los docentes acerca de las características de sus estudiantes, destacaban su condición socioeconómica, a lo que se añadía generalmente una adjudicación de carencias afectivas que conseguían ensamblarse a una explicación de la acrecentada violencia percibida en sus modos de relacionarse.

A lo largo del año 2013 llevamos a cabo observaciones participantes de jornadas escolares completas de manera periódica (dos veces a la semana al comienzo de nuestra inserción en el campo y luego semanalmente) construyendo registros etnográficos. Esta clase de registros da relevancia a la capacidad del investigador para observar y participar generando relatos escritos sobre lo vivenciado y acontecido. Un ejercicio en que la práctica descriptiva no se escinde de la interpretativa, por lo que resulta necesario distinguir entre lo que los actores dicen o hacen y las interpretaciones del investigador (Gibbs, 2012). Esto supone identificar y recuperar los "términos nativos" (Valles, 1999), evitando un empleo precoz de la jerga de las ciencias sociales (Spradley, 2016). También realizamos entrevistas a estudiantes y docentes. Asimismo, el análisis de registros disciplinarios y académicos de los estudiantes nos permitió reconstruir algunos aspectos de la relación de los jóvenes con la escuela. El conjunto de materiales documentales y conversacionales fueron transcritos y digitalizados para conformar el corpus empírico sobre el cuál llevamos a cabo nuestros análisis.

Para la construcción de los datos partimos de una estrategia de análisis de contenido basada en una comparación constante (Glaser y Strauss, 1967) efectuada sobre la totalidad de nuestro corpus empírico. Dicha tarea -al no ser un momento escindido ni ulterior al trabajo de campo- estuvo guiada tanto por las preguntas de investigación como por las problemáticas e incidentes críticos que fueron emergiendo durante nuestra estadía en la escuela. Esto supuso una primera fase de familiarización con las rutinas cotidianas y, posteriormente, una atención enfocada hacia la comprensión de situaciones que, desde la perspectiva de los actores involucrados, resultaban disruptivas en algún aspecto. En la tarea analítica asumimos la pauta etnometodológica de acuerdo con la cual los acontecimientos disruptivos brindan oportunidad para comprender lo que ordinariamente se da-por-sentado en el mundo de la vida cotidiana (Garfinkel, 2006). Nuestra estrategia consistía en interrogar el material de campo, en busca de pistas esclarecedoras que permitieran ir construyendo hipótesis provisorias, poniéndolas a prueba en entrevistas y conversaciones cotidianas. En este sentido, las conversaciones sostenidas con nuestros informantes fungían como instancias de validación de nuestras construcciones analíticas. Asimismo, al concluir nuestra estadía en el campo, pudimos cotejar algunas de estas construcciones en reuniones con jóvenes y adultos de la escuela. 


\title{
El aula y su zonificación en la escuela Güemes
}

\author{
A la menor sugerencia de doble sentido sexual, surgen en \\ las filas de atrás risas y gritos, acompañados a veces de la \\ gesticulación de alguno que remeda la masturbación de un \\ pene gigantesco por encima de la cabeza, con gesto lascivo en \\ los labios apretados.
}

Willis (1988, p. 25).

Como en cualquier escuela, la cotidianidad de primer año transcurría en actividades grupales, charlas y juegos. Con frecuencia se transgredían algunas normas escolares y ello suscitaba la aparición de acciones docentes que buscaban restablecer el orden y recuperar el control de las clases. Atendiendo a su gravedad, dichas transgresiones podían ser objeto de advertencias, amenazas y coerciones, o documentadas a través de amonestaciones hacia quienes molestaran en lugar de trabajar:

Vilma, la docente de historia, se acerca y comienza a hablarme de Paula y de Miguel. La situación me incomoda porque Paula está escuchando lo que Vilma dice de ella: "Esta chiquita siempre está sola, yo la ubico en un grupo todas las clases y dura esa sola clase. Pero, a diferencia de aquel chiquito [Miguel] ella trabaja". Paula mira para abajo, parece avergonzada. Vilma prosigue: "En este curso están mezcladitos, por ejemplo: [señala con su dedo índice] ahí hay uno que trabaja con uno que molesta", dice señalando a Nehuén y Armando... (Observación de clase).

Intercalando comentarios dirigidos al investigador recién llegado al campo, Vilma continuaría recorriendo el aula con su mirada, clasificando en voz alta a los jóvenes según considerara que trabajasen o molestasen, aludiendo a lo que parecían ser rasgos estables de personalidad. Que la docente percibiera que los estudiantes de primer año estaban mezcladitos, nos informa acerca de su expectativa de hallarlos con cierta organización en la geografía del aula. En efecto, observamos que una de las intervenciones reguladoras más frecuentemente implementadas por los docentes buscaba incidir sobre dicha organización. Referimos a los cambios de lugar:

La docente se acerca a Nehuén y le pide que se siente adelante para que no moleste. Él se resiste. Ella insiste y amenaza: "Mando a buscar el libro de amonestaciones". Nehuén se mueve y dice: "Me siento acá", ubicándose en un banco del centro del aula. La docente continúa insistiendo hasta que él termina cediendo y se ubica en la primera fila de bancos. (Observación de clase) 
Un aspecto saliente de los cambios de lugar era que ocurrían en un solo sentido: como peones en un tablero del ajedrez, los jóvenes eran llevados desde la porción posterior del aula hacia la anterior, pero nunca en sentido contrario. Lo que parecía indicar que-como en el epígrafe que tomamos prestado a Paul Willis- los malos comportamientos también aquí ocurrían en las filas traseras. La figura 1 muestra un mapa del aula en el que puede apreciarse la disposición espacial de los jóvenes y su relación con los registros disciplinarios hacia la mitad del ciclo lectivo.

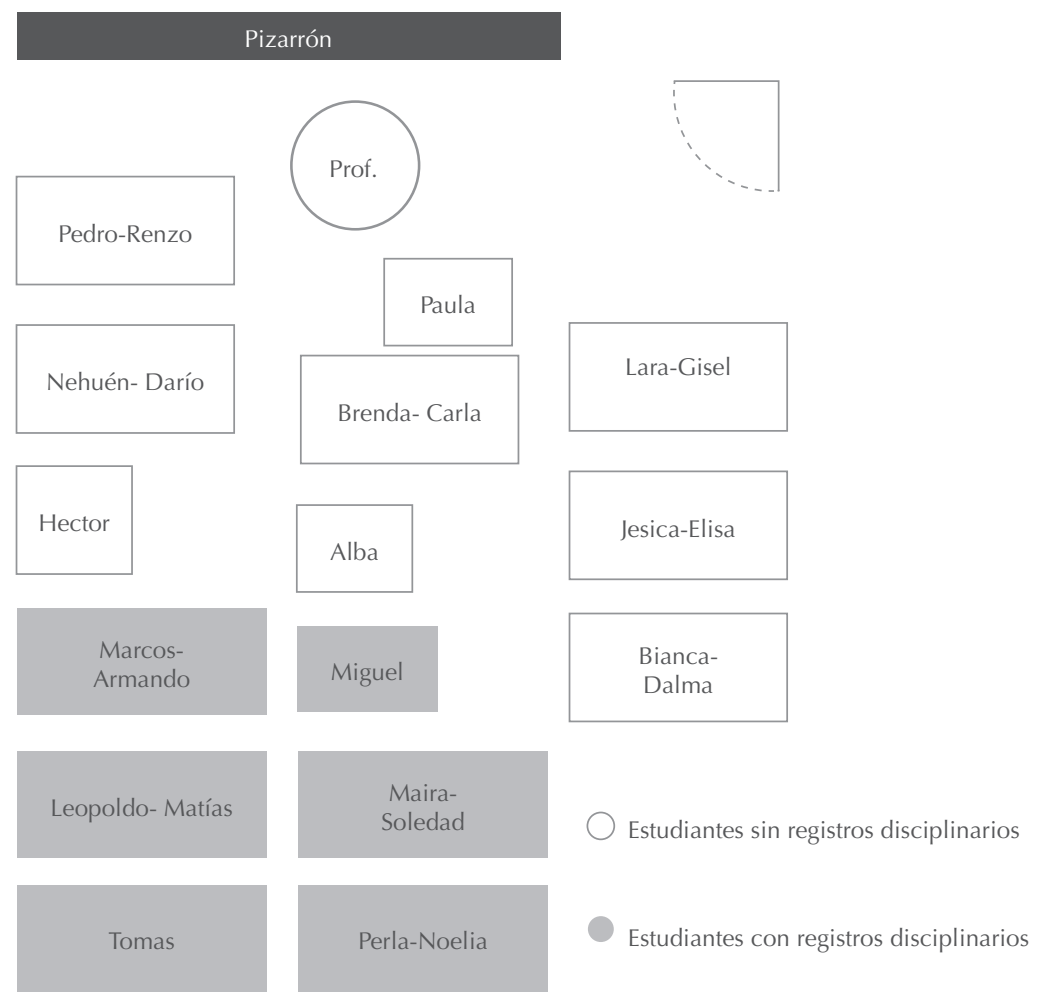

Figura 1. Distribución de los registros disciplinarios en el aula

Fuente: elaboración propia!.

1 El procedimiento para la construcción del mapa consistió en comparar las distribuciones de las aulas de primer año durante varias jornadas de observación, asignando a cada joven su lugar típico. El segundo paso incluyó la elaboración del plano de disposición y la asignación de marcas diferentes según los estudiantes registraran o no faltas disciplinarias. 
Resultaba difícil para nosotros no ver en este mapa una confirmación de que las posiciones en el aula se correspondían con formas determinadas de comportarse en la escuela (molestar o trabajar). Sin proponérselo, la profesora Susana fue la primera en advertirnos de nuestra acotada comprensión:

Susana: ... Me contaron de una alumna, que hoy la tengo en segundo año, que el año pasado estuvo haciendo sexo oral acá en la calle a cinco, seis chicos. Fue filmado [y difundido] por toda la escuela y ahora todo el curso le dice petera ${ }^{2}$ y le grita. $Y$ uno ve la chica y no parece que fuera ella [...] es una buena alumna, tranquila, es como no puedo creer que esa misma chica estaba haciendo eso. Nada que ver, es una chica que se sienta adelante, que es responsable... (Entrevista a Susana, docente)

Impactada por la situación que llegó a su conocimiento, Susana comentaba que la joven en cuestión se sentaba adelante. Presumía que con ello aportaba un dato para que comprendiéramos su perplejidad. Buena alumna, tranquila y sentada adelante eran rasgos que no lograba conciliar con lo que su estudiante había hecho en la calle. Ciertamente, la moralización del territorio del aula no era un aspecto inadvertido por los estudiantes. En sus entrevistas ellos explicaban que quienes se sentaban adelante procuraban que los profes los vieran, mostrando que querían estudiar. La fachada (Goffman, 1989) que se buscaba construir en la interacción con el docente era la de quien se sometía voluntariamente a un control más riguroso, como parte de una apuesta por el conocimiento. En cambio, la porción trasera del aula -por encontrarse alejada del docente- era un lugar propicio para joder y molestar. Formas de oposicionismo escolar que, tal como ha sido descrito en otras investigaciones, a veces buscaban conciliar el agobio o el aburrimiento producido por la escuela, con los intereses juveniles (Cerda y Assael, 1998; Saucedo, 2000). Aunque también, como se ha observado en numerosas oportunidades, parecía tratarse de las modalidades que asumía para ciertos varones la afirmación de una masculinidad hegemónica, activa y desafiante (Connell, 1997).

Que estas imputaciones referidas al espacio de aula proviniesen de jóvenes que recién comenzaban la secundaria, revelaba que estábamos ante aprendizajes que venían construyéndose en trayectos escolares previos. Pedro, de hecho, comentaba que, en la escuela primaria, cada vez que su maestra lo ubicaba adelante, él se sentía más vigilado y más solo. Darío se describía a sí mismo como el menos estudioso del grupo de los varones estudiosos. En el aula se ubicaba en una posición que él consideraba intermedia porque le permitía interactuar con los compañeros de adelante (presuntamente más estudiosos que él) y los ya consabidos molestadores del fondo. Un caso interesante era el de Renzo, que cursaba primer año por

2 En el lenguaje coloquial argentino, este término se utiliza como un insulto y designa despectivamente a una mujer que disfruta realizando sexo oral a varones. 
segunda vez. Empeñado en ser promovido en esta oportunidad, optaba por sentarse en la parte delantera del aula, reconociendo que sentarse atrás implicaba siempre el riesgo de verse involucrado en cosas. No obstante, aprovechaba los recreos y las clases de educación física para encontrarse con su grupo de amigos, que durante las clases se ubicaban atrás.

En todos los casos, nos encontramos ante estrategias de presentación de sí mismos (Goffman, 1989) que hacen un uso artefactual del espacio áulico al servicio de exhibir, situacionalmente, una adhesión a los aspectos normativos del rol estudiantil o, por el contrario, una distancia manifiesta. Esta clase de procesos de construcción de fachadas ha sido relevada por otras investigaciones en relación con pares de categorías, como bufones y payasos (Dubet y Martuccelli, 1998), colegas y pringaos (Willis, 1988), payasos y bien portados (Mejía Hernández, 2015).

Sin embargo, como veremos a continuación, otros elementos presentes en los testimonios juveniles ponían de manifiesto que los usos de la espacialidad no se agotaban en la conciliación de la tensión entre ser joven y estudiante. Asimismo, progresivamente fue haciéndose más claro para nosotros que los contornos de esta espacialidad no estaban definidos por las paredes del aula.

\section{Territorialidades urbanas y áulicas: de "la villa" al "fondo"}

Para indagar los sentidos que el territorio del aula tenía para los jóvenes, ingeniamos un ejercicio: en una serie de entrevistas entregamos a algunos de ellos un set de tarjetas que contenían los nombres de todos sus compañeros. Los jóvenes fueron invitados a participar atendiendo a su pertenencia a distintos grupos de afinidad, inferida por nosotros a partir de las observaciones participantes. Solicitamos que cada uno organizara las tarjetas para explicar cómo estaban conformados los grupos de amigos al interior del curso y dónde se ubicaban en el aula. Pese a los variados modos de entender la constitución y ubicación de estos grupos, Ilamó nuestra atención la nitidez con que los entrevistados identificaban uno en particular. Referimos al que conformaban cinco jóvenes (Perla, Soledad, Noelia, Maira y Tomás) que se sentaban al fondo del aula. Sus compañeros referían a ellos despectivamente como los brasas y negros.

En algunas entrevistas, el hito fundacional de este grupo era remitido a una jornada en la que dos de sus integrantes, Perla y Noelia, reproducían una canción del cantante de Cuarteto, "La Mona Jiménez" ${ }^{\text {"3 }}$ a través

3 Carlos "La Mona" Jiménez es el máximo referente del "Cuarteto". Este género musical nacido en Córdoba se caracteriza por un ritmo movido y alegre. Surge en los años 1940 como baile de la clase trabajadora, y en tanto tal, despreciado por la clase media y alta. 
del altavoz del teléfono celular de una de ellas. Bianca, otra compañera sentada un banco más adelante, giró sobre sí misma solicitando que no fueran tan brasas y que dejaran de escuchar esa música de negros. En su entrevista, Perla rememoraba el episodio comentando que desde hacía tiempo venía guardando muchas cosas y que ese día sintió que no aguantaba más. Esperó hasta la finalización de la jornada y una vez fuera del colegio agredió físicamente a Bianca, golpeándola y tirándole el pelo:

Brenda: A principio de año Bianca se sentaba al último.

E: ¿Por qué se cambió de lugar Bianca? ¿Tienen idea?

Gisel: Porque a Bianca le caía mal Perla y decía que era una negra, entonces se pelearon.

Jésica: O sea, todo ese grupo [los brasas] la discriminaban a Bianca porque tenía ojos verdes, por cómo se pintaba [maquillaba], porque usaba ropa de marca...

Brenda: ... Y Bianca la discriminaba a Perla porque decía que escuchaba música de negros.

E: ¿Qué es ser negro?

Brenda: Cuando escuchan a La Mona. La manera de vestir... (Entrevista a Brenda y Jésica, estudiantes)

Tal como fue identificado también en otros trabajos cordobeses (Maldonado, 2006; Paulín, 2014; Tomasini, Bertarelli y Morales, 2017), algunos de los artefactos identitarios en que ancla la descripción de una persona brasa o negra, remiten a las formas de hablar y vestirse. En el habla del brasa los jóvenes de nuestro caso de estudio identificaban un uso rutinario de vocablos locales como guacho, chorazo o culeado que, según explicaban -incluso los integrantes de esta minoría- constituyen indicadores de poca decencia. Su vestimenta era definida por oposición a la del cheto, par antagónico del brasa; el primero, ajustado a la descripción que en la cita previa se hacía de Bianca, viste ropas de marca de colores sobrios. El segundo, parece tener debilidad por la ropa fosforescente y el calzado deportivo y voluminoso. Por último, el brasa escucha música de Cuarteto, mientras que el cheto prefiere música electrónica o pop, con letras en inglés.

La detallada caracterización estética que se obtiene preguntando a los jóvenes por el significado de este par de categorías, difícilmente evite la impresión que el lector debe haberse forjado: que la discriminación hacia quién tenía ojos verdes en un contexto en el cual -vale decirlo- es un

Aunque esta situación cambia desde los años 1990, cuando el género empieza a tener pregnancia en distintos sectores sociales en Argentina. 
color inusual, acompañada de la acusación de negros hacia quienes aquí tenían tez morena, nos informa que el prejuicio racializado era un artefacto más en escena ${ }^{4}$ :

Brenda: A ellos no les molesta que les digamos brasas. Igual nadie se los dice, porque si les decimos le acusan a la preceptora...

Jésica: ¡Noooo! Aparte después te esperan a la salida [de la escuela], van y te pegan... (Entrevista a Brenda y Jésica, estudiantes)

Está claro que decir de alguien que es brasa implica realizar un juicio peyorativo. No ocurre lo mismo con el cheto. Incluso cuando la categoría puede ser usada despectivamente, su indumentaria y los consumos culturales se asocian a cierto poder adquisitivo y un sentido estético que trasciende lo local (la ropa de marca, la música anglosajona). De hecho, notamos que quienes se adjudicaban ante nosotros el taxón de normales (ni brasas, ni chetos) certificaban su normalidad exaltando rasgos personales más próximos a la caracterización del cheto. Elías (1998) y Goffman (2006) nos enseñaron que un atributo descalificador solamente consigue expresarse en un lenguaje de relaciones ordenadas por diferenciales de poder. No obstante, en el fragmento previo, Brenda nos conectaba con un aspecto singular de nuestro caso de estudio, que nuestra familiaridad previa con la categoría de brasas y su carácter desdeñoso haría que tardásemos en comprender. Para estos cinco jóvenes la categoría nombraba atributos identitarios que consolidaban cierta estima de sí:

Perla: Somos como tipo brasas. En el grupo nosotros somos todos así y a mí me gusta estar con ellos porque son iguales que yo. En cambio, con ellas [las chicas de otros barrios] no me gusta estar mucho porque son de otra forma, no son igual que yo, entonces no me gusta mucho. Porque te están diciendo así, que seas de otra forma, de todo te dicen. Mi mamá me dice que soy mal hablada, no le gusta que hable así porque soy como más de La Villa, a ella no le gusta eso.

E: Pero ¿dónde aprendiste a hablar así?

4 En Argentina, como apuntó Margulis (1999), el racismo no está anclado a la idea de raza exclusivamente sino a la descalificación, inferiorización y exclusión de grupos por distintas razones. Lo negro no se asocia necesariamente a rasgos fenotípicos, sino que se aplica a una población heterogénea que vive en las villas o a personas de sectores populares o en función de sus prácticas y consumos culturales, sus estéticas y estilos corporales que se devalúan por medio de tal calificación. Mediante categorías racializadas como negros o negros brasas se inferioriza, devalúa o discrimina a las personas, en estrecha relación con la clase social. No obstante, tal como lo analizó Mónica Maldonado (2006) en la década de 1990, el prejuicio racializado no se genera solo a partir de los sectores hegemónicos o dominantes. Frecuentemente, los grupos más desfavorecidos retoman y amplifican los prejuicios para acortar las distancias con los sectores dominantes. 
Perla: De ahí de mi barrio, me empecé a juntar con los chicos y me gustó cómo hablaban ellos. Tengo muchos amigos que hablan así, entonces se me pegó a mí hablar así.

E: Y en los barrios donde viven estas chicas: ¿no se habla así?

Perla: No, son como más chetitos, hablan de otra forma, como más decente, algo así.

E: ¿Más decente?

Perla: Sí, así me dice mi mamá: que tengo que ser como ellos. Pero a mí no me gusta la forma de ser de ellos. (Entrevista a Perla, estudiante)

A pesar de la opinión de su madre (que en su deseo de lo que Perla debería ser, conseguía posicionarla como lo que efectivamente era: poco decente) la joven nos explicaba que ser brasa era algo que la identificaba con sus amigos del barrio. Hacía aparecer un elemento relevante que hasta el momento no habíamos advertido: en este caso de estudio, detrás de las categorías sociales de brasas y chetos —-taxonomías cuyo significado y circulación se extienden más allá del ámbito de esta escuela— se ocultaba el dato de la procedencia barrial de estos cinco jóvenes:

E: ¿Vos sabés por qué motivo se pelearon Bianca y Perla?

Renzo: Por la forma de ser, porque Bianca le decía a Perla que era brasa, por la música que escuchaba. Sí, digamos... por el barrio. Ellos cinco [los brasas] son de Villa El Libertador. Yo también soy de ahí pero... soy del principio [del barrio]. El fondo, donde ya está terminando Villa El Libertador, ahí ya son todos... roban, se juntan en bandas... (Entrevista a Renzo, estudiante)

Al igual que Renzo, varios jóvenes de este curso provenían de Villa El Libertador, uno de los barrios populares más importantes de la zona sur de Córdoba. Este conocimiento nos condujo a desestimar inicialmente su importancia en los procesos de diferenciación intergrupal. No obstante, los brasas venían del fondo del barrio -región de aparente reputación peligrosa- para ubicarse en el fondo del aula. Si decíamos líneas más arriba que para algunos jóvenes las filas traseras eran una región estratégica para molestar, Perla y Noelia aducían en una conversación con nosotros que la ubicación escogida respondía a una necesidad de ocultarse del resto de sus compañeros: "Para que los demás no nos vean tanto" escolar, la preceptora del curso ordenó a estas jóvenes que se sentaran adelante y recomendó alejarse de sus otros tres amigos, augurando que las llevarían por mal camino. Posiblemente, la docente se refiriere a que Tomás,

5 Ciertamente, su estrategia no difería demasiado de la nuestra; la opción por el fondo del curso también permitía al investigador ocultarse, ser visible para menos estudiantes y llamar menos la atención de los docentes. 
Soledad y Maira eran estudiantes repetidores. Lo cierto es que por motivos diferentes, Lara y Gisel (alumnas de la primera fila) parecían acordar con este enjuiciamiento:

Me acerco a Lara y le pregunto por los grupos de amigos que hay en el curso. A los del fondo les Ilama los barderos, porque gritan y hacen lío. En ese momento pasan cerca de nosotros Maira y Soledad jugando a empujarse, riéndose a carcajadas. Con expresión confirmatoria y algo despectiva, Lara me dice: “¿vio?". (Observación de clase)

Gisel: Acá atrás se sientan los brasas [dice señalando un mapa imaginario del aula que demarca en la mesa].

E: ¿Para vos quiénes serían brasas en el curso?

Gisel: Tomás... no es tan quilombero pero es mal hablado y si te juntás con él es obvio que vas a hablar mal... (Entrevista a Gisel, estudiante)

En las conversaciones anteriores, barderos y quilomberos eran los calificativos que Lara y Gisel hacían aparecer al hablar de este grupo de jóvenes. En Argentina, bardo y quilombo son modismos que significan "desorden" o "problema". Bardero o quilombero es alguien que se comporta de manera problemática. Algunos usos de estas categorías amplían su significado para abarcar la comisión de actos delictivos o violentos (Di Napoli, 2013; Kessler, 2007) aunque también en el contexto escolar son términos que se ajustan para designar a quienes estorban el orden disciplinario (García Bastán, 2015). Se trata de categorías que, al no pertenecer a un mundo figurado en particular, asumen su significado de modo indexical (Garfinkel, 2006) ${ }^{6}$. En este contexto, aparecen valorando ciertas prácticas (usos corporales, formas de hablar o de comportarse) y consiguen yuxtaponerse a otras categorías de significado próximo, propias del mundo escolar en que los jóvenes están siendo socializados (molestar, portarse mal, ser el peor del curso). Así, con el correr de las jornadas escolares, la condición estético-barrial de brasas atribuida a estos cinco jóvenes, enlazada a la de barderos y quilomberos, parecía adherirse a ciertas identidades escolares posicionales:

Tomás: [Los profesores] siempre nos miran a nosotros, a los del fondo nomás, y no somos siempre nosotros los que hacemos las cosas, ¿me entendés?

E: ¿Por qué te parece que los miran a ustedes entonces?

Tomás: Y... porque somos los peores del curso.

E: ¿En qué sentido?

6 En la tradición de la etnometodología, la indexicalidad o indicialidad refiere al carácter contextual del lenguaje que hace que su inteligibilidad deba encontrarse en la propia situación de interacción. 
Tomás: En que nos portamos mal. Pero a veces no somos nosotros y nos miran a nosotros cinco por la forma de hablar para mí, por el barrio. En cambio, los otros no insultan, bah... insultan pero a veces los profesores se hacen los que no escuchan.

E: ¿Cómo es esto del barrio?

Tomás: Y... yo vivo en la Villa. (Entrevista a Tomás, estudiante)

Tomás entendía que la condición escolar de su grupo (el hecho de saberse catalogados por los docentes como los peores del curso) era un deslizamiento de su procedencia barrial. El dato de que en este curso otros jóvenes habían recibido más sanciones disciplinarias que ellos, bastaría para echar por tierra su argumento. Sin embargo, este cobra sentido al integrarlo en una cadena de episodios de posicionamiento social. La pertenencia barrial que estos cinco jóvenes comparten (el fondo del barrio, donde la gente roba) y su aspecto moreno, posiblemente hayan sido los primeros artefactos de la serie que los conduciría a constituirse como los peores del curso. Si bien el material presentado muestra que la actitud de estos jóvenes frente a las prácticas de posicionamiento dirigidas hacia ellos no fue pasiva ni conformista, su reclusión hacia las filas traseras del aula confirmaba, ante compañeros y docentes, que eran jóvenes que querían molestar, se portaban mal e iban por mal camino. De modo similar, la reacción de Perla ante el posicionamiento impuesto por Bianca (enfrentarla afuera de la escuela), acrecentó su vulnerabilidad ante las etiquetas que ella y sus amigos habían ido colectando. Reafirmando a ojos de sus compañeros que efectivamente portaba actitudes de bardera, propias de la región del barrio de la que procedía.

La intervención de la escuela ante este incidente también revela espacialidades y prácticas de posicionamiento. Por haber ocurrido fuera del edificio escolar, las jóvenes involucradas no recibieron apercibimientos disciplinarios. El mensaje implícito es contundente: solo incumbe a la escuela lo que suceda puertas adentro. Por otro lado, la escuela actuó de oficio, señalando a Perla como la iniciadora del conflicto en el registro que, como recaudo ante un eventual pedido de explicaciones por parte de los padres, su preceptora escribió en el cuaderno de seguimiento de primer año ${ }^{7}$. Así, simultáneamente, el establecimiento eludía la responsabilidad por lo sucedido y practicaba un posicionamiento hacia las jóvenes en clave de víctima/victimaria. Perla produjo un acto que le valdría una nueva capa a la ya espesa identidad de su grupo de amigos. La espectacularidad de su

7 El "cuaderno de seguimiento" era un registro informal que llevaban los preceptores de cada curso. A pedido de la gestión directiva, todo lo que sucediese a los jóvenes debía quedar registrado allí. Especialmente, para poder mostrar a los padres que se acercaran a la escuela con algún reclamo que existía un seguimiento riguroso de la trayectoria de sus hijos. 
reacción y la intervención escolar, consiguieron opacar un trayecto previo en el que la joven decía venir aguantando silenciosamente una serie de injurias discriminatorias. La pelea afuera es el artefacto que sanciona el nuevo episodio de posicionamiento, que se integra a la serie preexistente:

E: Veo que los varones se pelean mucho adentro de la escuela y a las chicas no las veo pelear tanto. Pero me llama la atención que [desde que estoy en la escuela] hubo dos peleas [entre chicas] afuera...

Perla: Para mí puede ser [que las chicas se peleen afuera] para que no les pongan amonestaciones o las echen del colegio. Pero en mi caso no fue por eso, sino porque no aguanté más y me quise sacar la bronca [Enojo]. Porque casi todas las peleas de chicas son afuera del colegio.

E: Antes de llegar a pelearte con Bianca: ¿No habías hablado con nadie acá [en la escuela]?

Perla: Con mi grupo nomás.

E: ¿Y qué te decían?

Perla: Que la deje, que no le dé bola [que no le dé importancia a lo que dice]. Pero después no di más y se me explotaron todos los nervios. (Entrevista a Perla, estudiante)

Teniendo en cuenta, como señalábamos al introducir este escrito, que el espacio no debe separarse analíticamente de las prácticas, el afuera escolar, culturalmente consolidado como locus de las peleas de chicas, aparece como el lugar lógico donde tramitar un conflicto que la escuela no considera de su incumbencia.

Hasta aquí, nuestros análisis encuentran similitudes con los hallazgos de otros estudios argentinos. En la provincia de Buenos Aires se han señalado ya las equivalencias que en la escuela pueden establecerse entre taxonomías sociales y escolares (Kaplan, 2008; Kaplan y Di Napoli, 2017; Mutchinick y Silva, 2013). Sin embargo, estos trabajos confluyen pensando que el poder simbólico de los actos de clasificación escolar traduce las categorías sociales en escolares, con lo cual el proceso parecería ocurrir con cierta linealidad. También en Córdoba algunas investigaciones han mostrado que en ámbitos escolares y barriales los significados y usos de la categoría de brasas se articulan a lo que Belvedere (2002) Ilamó una lógica elusiva de la discriminación, donde la jerarquización de unos es construida mediante la desvalorización de otros. Brasas, negros y villeros aparecen como categorías que permiten, situacionalmente, demarcar una distancia social y moral con el otro (García Bastán y Paulín, 2016; Maldonado, 2006; Paulín, 2015), o asumir una fachada temible para protegerse o defenderse (Tomasini, Bertarelli y Morales, 2017). Aunque nuevamente aquí un matiz merece ser destacado. A diferencia de lo que muestran los trabajos citados, los jóvenes segregados en nuestro estudio no intentaban 
desmarcarse de la identidad atribuida. Se decían brasas, disfrutaban escuchando Cuarteto públicamente en los espacios escolares y hablaban de un modo que sabían indecente. Incluso frente a lo que Perla reconocía como un mandato de su madre, ella se mostraba decidida a continuar comportándose como brasa. Tal como sugería Brenda, lo que parecía molestar a los integrantes de este grupo no era ser identificados como brasas, sino el menosprecio asociado a la marcación. Sin proponérselo, estaban disputando el estatus moral de la categoría.

Una mirada presta a los determinismos pensaría que estos jóvenes fueron arrastrados por una sucesión de posicionamientos que los condujeron a asumir una identidad devaluada y progresivamente estabilizada. Esa parecía ser la lectura reflexiva de Tomás, cuando renegaba de la injusta consideración que el grupo recibía de sus profesores por el barrio en que vivían. Sin embargo, una lectura menos monolítica es posible a medida que enfocamos procesos y trayectorias cada vez más singulares.

A diferencia de Tomás, que ese año tuvo uno de los peores desempeños de la clase, Perla logró cumplir exitosamente con las exigencias académicas. El enfrentamiento físico con Bianca consiguió que sus compañeras de otros barrios dejaran de molestar a su grupo por ser brasas. Además, pelear afuera implicó un manejo artefactual de espacios y tiempos, que la libraron de las consecuencias disciplinarias que su acto hubiese tenido dentro de la escuela. La joven sostuvo un posicionamiento identitario a pesar de las devaluadas posiciones de sujeto ofrecidas por la escuela, sus compañeros y su propia madre. Obrando incluso en contra de los consejos de ignorar a Bianca que le habían dado sus amigos. Perla se apropió críticamente de los artefactos culturales disponibles. Sacó considerable provecho de la situación en que se encontraba al asumir un rol protagónico en su constitución identitaria que podemos comprender como parte de una lógica de subjetivación (Dubet, 2010).

Esta lectura no implica caer en una mirada romántica ni celebratoria de episodios violentos, como la pelea a la que referimos, ni tampoco desconoce que haya existido un proceso discriminatorio evidente hacia este grupo de jóvenes. Contrariamente, consideramos que este proceso se produjo como resultado de una ausencia de acciones escolares planificadas con el objetivo de deconstruir las caracterizaciones estigmatizantes en el espacio de sociabilidad juvenil.

Como apuntamos anteriormente, algunos trabajos latinoamericanos señalan que conocer al otro diferente es un ingrediente sustancial en la vida de los jóvenes y que en ese proceso la violencia puede constituir una herramienta para el conocimiento de ese otro y la construcción de reconocimiento. Sin embargo, las interacciones horizontales en la escuela también han sido valoradas en su potencialidad para la integración de minorías sociales (Vásquez y Martínez, 1996). Así, las intervenciones escolares

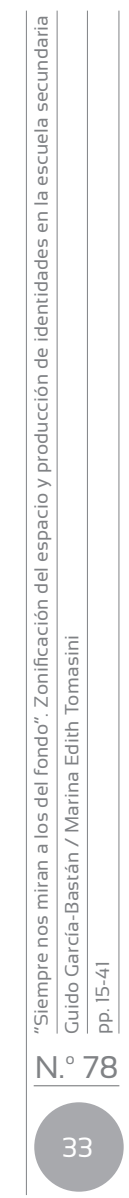


(por acción u omisión) y las prácticas de sociabilidad juvenil, a menudo consiguen un mutuo acople que apuntala procesos de estigmatización. Pero dichas intervenciones podrían también orientarse de modo sistemático a tratar con estos mismos procesos desde un paradigma de reconocimiento y respeto por la diversidad, en el marco de acciones que promuevan la convivencia en las escuelas. Para terminar este escrito reflexionado sobre las prácticas educativas, permítasenos reproducir un último testimonio. El de la docente de geografía refiriendo al grupo del fondo:

Viviana: Estos cinco, me pueden [...] Me costaría ponerles un límite. [...] Es como que manejan otros códigos, no sé cómo explicarte, pero vos cuando estás ahí te das cuenta, es como si quedara algo flotando, no te puedo explicar.

E: Y esa sensación: ¿Qué te produce? ¿Cómo te hace sentir?

Viviana: En un momento incómoda, porque yo reconozco que hay cosas que a ellos les permito y que a otros no. Por ahí los veo en una situación tan de desventaja... vos te das cuenta de que hay una necesidad. [...] Por ahí me cuestiono y digo: "Me llegan a ver los que me formaron y me sacan el título". (Entrevista a Viviana, docente)

La sensación de que los jóvenes del fondo la pueden y la incomodidad que le produce reconocer que el trato hacia ellos no es completamente imparcial, son indicadores de que Viviana incumplía ciertas expectativas personales referidas al rol docente. Sin embargo, fue la única docente entrevistada que pudo objetivar que en la sociabilidad de primer año algo quedaba flotando en relación con estos cinco, comprendiendo que eran necesarias otras condiciones para poder acogerlos en la escuela. La marcada homogeneidad social que solemos suponer en las escuelas, sumada al mandato ético racionalista que exige el trato ecuánime bajo la premisa de que todos somos iguales, puede conducirnos a desconocer la diversidad social y cultural que alojan nuestras escuelas, distanciándonos de una ética de la responsabilidad y el cuidado (Haynes, 2002). Dando lugar a prácticas que (por acción u omisión) pueden producir que algunos jóvenes, como Tomás, consideren que la escuela los discrimina por sus rasgos culturales, y que otros docentes, como Viviana, sientan culpa por mostrar preocupación y contemplación ante situaciones singulares de sus estudiantes.

\section{Reflexiones finales}

Nuestro punto de partida en este trabajo fue un entendimiento del espacio como dimensión analíticamente inseparable de las prácticas sociales. 
capacidad de generar posicionamientos identitarios al mostrar cómo la zonificación del espacio de aula, escolar y barrial se tornó en un importante artefacto identitario, mediando activamente en la estabilización de la identidad social y escolar de un grupo de estudiantes.

Hubiese sido imposible atender a estos procesos sin suspender una noción del aula y la escuela como recipientes (Leander et al., 2010). No se trata solo de un discurso dominante que prevalece en muchas aproximaciones investigativas, es también una narrativa activamente fomentada por la cultura de la organización escolar. En el caso analizado, esto se hizo palpable en la máxima femenina de pelear afuera que Perla evocaba en su entrevista. Cerrada sobre sí misma, la escuela consigue diferir este tipo de interacciones en mayor medida que disuadirlas. Así, la construcción discursiva de la escuela como recipiente pacificado no es más que una lectura deficitaria, incapaz de mirar el espacio escolar en su imbricación y sus nexos con espacios y procesos que exceden su perímetro.

La fertilidad de esta clave de lectura se hizo particularmente clara en el caso analizado. El proceso socializador escolar consiguió que algunas diferencias de los estudiantes quedaran adheridas a categorías y posicionamientos escolares, contribuyendo a ratificar en el espacio de sociabilidad juvenil el valor de categorías de génesis no escolar (negros, brasas), que en este caso se encontraban fuertemente vinculadas con la procedencia barrial de algunos jóvenes. Sin embargo, procuramos enfatizar que este proceso no fue mecánico ni lineal. En él intervinieron instrumentos simbólicos, espacio-temporales y corporales, cuyo reconocimiento nos permitió arribar a una lectura compleja, matizada y contemplativa de la agencia de los actores para operar sobre sus mundos figurados. Lo que mostramos a partir de este caso de estudio es una manera singular por la cual se conjugaron diversos artefactos (espaciales, temporales, barriales, estéticos, escolares) en los procesos de constitución identitaria, ensamblándose al complejo interjuego entre los procesos de socialización escolar y sociabilidad juvenil

Consideramos haber contribuido a mostrar el potencial que la comprensión de la sociabilidad juvenil aloja como herramienta para la intervención en las escuelas. La ajenidad y falta de comprensión con la que muchas veces se tratan las problemáticas juveniles, comporta un riesgo permanente de reforzar con artefactos escolares procesos discriminatorios que corresponden a mundos figurados más abarcativos. El desafío para las escuelas parece ser el de intervenir oportunamente; prestar atención a los recursos culturales que movilizan los estudiantes, tanto como a los que la escuela tiene para ofrecer; proponer marcos de inteligibilidad que posibiliten el reconocimiento genuino de la diversidad cultural en las prácticas educativas. 
Por último, nuestros resultados en absoluto agotan la complejidad de los fenómenos de posicionamiento espacial en la escuela. Es necesario continuar explorando las implicancias afectivas, psicológicas y sociales que las prácticas de posicionamiento, como las que analizamos aquí, tienen para la constitución del self. Asimismo, debido al modo en que construimos nuestros interrogantes iniciales, las observaciones realizadas priorizaron el contexto de la clase. La posibilidad de pensar el espacio de aula en sus imbricaciones con otros espacios escolares y extraescolares fue un aspecto emergente en nuestra investigación. En ese sentido, reconocemos que otro plano de la espacialidad que requeriría explorarse es aquel que puede congregar a otros jóvenes de la comunidad escolar que no pertenezcan al mismo curso pero puedan, eventualmente, compartir la procedencia barrial u otras referencias geográficas e identitarias. Referimos a niveles intermedios de la geografía escolar que no responden necesariamente a las secuencias institucionales: recreos, encuentros en los pasillos, salidas anticipadas de clase. Seguramente allí encontraremos otro abanico de prácticas de posicionamiento a partir de las cuales se construyen identidades juveniles.

\section{Referencias}

Álvarez Uría, F. y Varela, J. (1991). Arqueología de la escuela. Madrid: La Piqueta.

Barrett, P., Davies, F., Zhang, Y. y Barrett, L. (2015). The impact of classroom design on pupils' learning: Final results. Building and Environment, 89, 118-133. Dol: https://doi.org/10.1016/j.buildenv.2015.02.013

Beer, D. (2008). Visiones y discursos en la Educación Física de la escuela. En G. Morgade y G. Alonso (comps.), Cuerpos y sexualidades en la escuela (pp. 149-173). Buenos Aires: Paidós.

Belvedere, C. (2002). De sapos y cocodrilos. La lógica elusiva de la discriminación social. Buenos Aires: Biblos.

Berger, P. y Luckmann, T. (2001). La construcción social de la realidad. Buenos Aires: Amorrortu.

Brito Rivera, L.F., Subero Tomás, D. y Esteban-Guitart, M. (2018). Fondos de conocimiento e identidad: Una vía sociocultural de continuidad educativa. Revista Educación, 42(1), 1-23. Dol: https://doi.org/10.15517/ revedu.v42i1.23470

Cerda, A.M. y Assael, J. (1998). Normatividad escolar y construcción de valores en la vida cotidiana del liceo. Perspectivas, 28(4), 629-644.

Connell, R. (1997). La organización social de la masculinidad. En T. Valdés y J. Olavarría (eds.). Masculinidades. Poder y crisis (pp. 31-48). Santiago de Chile: Isis Internacional. 
Di Napoli, P. (2013). Violencia, racismo y escuela. El caso de los alumnos tipificados como violentos. Propuesta educativa, 22(39), 43-50. Recuperado de http://propuestaeducativa.flacso.org.ar/archivos/jovenes/18.pdf

Dubet, F. (2010). Sociología de la experiencia. Madrid: Editorial Complutense y cIS.

Dubet, F. y Martuccelli, D. (1998). En la escuela. Sociología de la experiencia escolar. Buenos Aires: Losada.

Dussel, I. y Caruso, M. (1999). La invención del aula. Una genealogía de las formas de enseñar. Buenos Aires: Santillana.

Elias, N. (1998). Ensayo teórico sobre las relaciones entre establecidos y marginados. En N. Elias. La civilización de los padres y otros ensayos (pp. 79-138). Bogotá: Norma.

Esteban-Guitart, M. y Moll, L. C. (2014). Funds of identity: A new concept based on the funds of knowledge approach. Culture \& Psychology, 20(1), 31-48. DOI: https://doi.org/10.1177/1354067×13515934

Evertson, C. y Weinstein, C. (2011). Handbook of Classroom Management. Research, practice and contemporary issues. Nueva York: Routledge.

Foucault, M. (2008). Vigilar y castigar. Nacimiento de la prisión. Buenos Aires: Siglo xxı.

García Bastán, G. (2015). "Quilomberos... pero bien educados": La buena educación como competencia moral de sectores de élite. Un estudio de caso sobre una escuela secundaria de la ciudad de Córdoba, Argentina. Revista Brasileira de Sociologia da Emoção, 14(40), 64-78. Recuperado de http://www.cchla.ufpb.br/rbse/RBSE\%20v.14,\%20 n.40,\%20abril\%202015\%20completa.pdf\#page=60

García Bastán, G. (2017). Evaluación informal y conflictividad en la escuela media: sentidos y prácticas de docentes y alumnos en sus interacciones. [Tesis doctoral]. Facultad de Psicología, Universidad Nacional de Córdoba, Córdoba.

García Bastán, G. y Paulín, H.L. (2016). Identidades juveniles en escenarios de periferización urbana: una aproximación biográfica. Quaderns de Psicología, 18(1), 35-52. Dol: https://doi.org/10.5565/rev/qpsicologia. 1307

García, M. y Madriaza, P. (2005). Sentido y sinsentido de la violencia escolar: análisis cualitativo del discurso de estudiantes chilenos. Psykhe, 14(1), 165-180. DOI: https://doi.org/10.4067/s0718-22282005000100013

Garfinkel, H. (2006). Estudios en etnometodología. Barcelona: Anthropos.

Gibbs, G (2012). El análisis de datos en investigación cualitativa. Madrid, España: Morata.

Giddens, A. (1984). The Constitution of Society. Outline of the Theory of Structuration. Cambridge: Polity Press. 
Glaser, B. y Strauss, A. (1967). The discovery of grounded theory. Strategies of qualitative research. Nueva York: Aldine.

Goffman, E. (1983). The interaction order. American Sociological Review, 1(48), 1-17.

Goffman, E. (1989). La presentación de la persona en la vida cotidiana. Buenos Aires: Amorrortu.

Goffman, E. (2006). Estigma. La identidad deteriorada. Buenos Aires: Amorrortu.

Gómez Cabrera, A. P. (2016). Representación, significado e interacción en el acoso escolar juvenil. Revista Científica Guillermo de Ockham, 14(2), 1-23. Recuperado de https://revistas.usb.edu.co/index.php/ GuillermoOckham/article/view/2426/2142

Haynes, F. (2002). Ética y escuela ¿Es siempre ético cumplir las normas de la escuela? Barcelona: Gedisa.

Holland, D. y Leander, K. (2004). Ethnographic Studies of Positioning and Subjectivity: An introduction. Ethos, 32(2), 127-139. Dol: https://doi. org/10.1525/eth.2004.32.2.127

Holland, D., Lachicotte, W., Skinner, D. y Cain, C. (1998). Identity and Agency in Cultural Worlds. Cambridge: Harvard University Press.

Ibáñez, T. (1992). La tensión esencial de la psicología social. En D. Páez et al. (eds.), Teoría y método en Psicología Social (pp. 13-28). Barcelona: Anthropos.

Kaplan, C. (2008). Talentos, dones e inteligencias. Buenos Aires: Ediciones Colihue.

Kaplan, C. y Di Napoli, P. (2017). Tipificaciones juveniles sobre la violencia en el escenario escolar. Ultima década, 25(46), 147-183. DOI: https://doi.org/10.4067/s0718-22362017000100147

Kessler, G. (2007). Escuela y delito juvenil. La experiencia educativa de jóvenes en conflicto con la ley. Revista Mexicana de Investigación Educativa, 12(32), 283-303. Recuperado de http://www.scielo.org. $\mathrm{mx} / \mathrm{pdf} / \mathrm{rmie} / \mathrm{v} 12 \mathrm{n32/1405-6666-rmie-12-32-283.pdf}$

Leander, K. (2002). Locating Latanya: the situated production of identity artifacts in classroom interaction. Research in the Teaching of English, 37(2), 198-250. Recuperado de https://www.jstor.org/stable/40171622?seq=1\#page_scan_tab_contents

Leander, K., Phillips, N. y Headrick Taylor, K. (2010). The Changing Social Spaces of Learning: Mapping New Mobilities. Review of Research in Education, 34, 329-394. Dol: https://doi.org/10.3102/0091732x09358129

Lefebvre, H. (1991). The Production of Space. Oxford: Blackwell.

Maffesoli, M. (1990). El tiempo de las tribus. Barcelona: Icaria. 
Maldonado, M. (2006). Una escuela adentro de una escuela. Un enfoque antropológico sobre los estudiantes secundarios en una escuela pública de los '90. Buenos Aires: Eudeba.

Margulis, M. (1999). La "racialización" de las relaciones de clase. En M. Margulis y M. Urresti (coords.), La segregación negada: Cultura y discriminación social (pp. 37-73). Buenos Aires: Biblos.

Mejía-Hernández, J.M. (2015). Del "Payaso" al "chavo bien portado". Algunas "formas de ser adolescente" entre varones de secundaria. Revista Mexicana de Investigación Educativa, 20(67), 1081-1104. DOI: https://www.redalyc.org/pdf/140/14042022004.pdf

Mejía Hernández, J.M. y Weiss, E. (2011). La violencia entre chicas de secundaria. Revista Mexicana de Investigación Educativa, 16(49), 545-570. Recuperado de http://www.comie.org.mx/documentos/ rmie/v16/n049/pdf/49009.pdf

Mutchinick, A. y Silva, V. (2013). Las relaciones de humillación y la construcción de la autoestima escolar. La mirada de los jóvenes estudiantes sobre los sentimientos de superioridad e inferioridad. En C. Kaplan (dir.), Culturas estudiantiles. Sociología de los vínculos en la escuela (pp. 69-102). Buenos Aires: Miño y Dávila.

Narodowski, M. (2008). Infancia y poder. La conformación de la pedagogía moderna. Buenos Aires: Aique.

Nash, C. (1981). The Effects of Classroom Spatial Organisation on Four- and Five-Year-Old Children's Learning. British Journal of Educational Psychology, 51(2), 144-155. Dol: https://doi.org/10.1111/j.2044-8279.1981. tb02468.x

Paulín, H. (2014). "Gente negra" y "gente cheta" en la escuela: disputas por el reconocimiento en estudiantes secundarios de la ciudad de Córdoba, Argentina. Cuadernos de Educación, 12(12), 1-14. Recuperado de https://revistas.unc.edu.ar/index.php/Cuadernos/article/view/9222

Paulín, H. (2015). "Ganarse el respeto". Un análisis de los conflictos de la sociabilidad juvenil en la escuela secundaria. Revista Mexicana de Investigación Educativa, 20(67), 1105-1130. Recuperado de http://www.scielo.org.mx/scielo.php?script=sci_arttext\&pi$\mathrm{d}=$ S1405-66662015000400005

Paulín, H.L. y Tomasini, M. (2007). Inducción, apropiación y resistencia. Discusiones sobre el concepto de socialización en las prácticas educativas. Revista Conciencia Social, 10, 67-74.

Puiggrós, A. (1990). Sujetos, disciplina y currículo en los orígenes del sistema educativo argentino. Buenos Aires: Editorial Galerna.

Rist, R. (1970). Student social class and Teacher expectations: The self-fullfilling profecy in ghetto education. Harvard Educational Review, 40(3), 411-451. DOI: https://doi.org/10.17763/haer.40.3.h0m026p670k618q3 
Rosenfield, P., Lambert, N. y Black, A. (1985). Desk arrangement effects on pupil classroom behavior. Journal of Educational Psychology, 77(1), 101-108. DOI: https://doi.org/10.1037/0022-0663.77.1.101

Saucedo Ramos, C.L. (2000). El "relajo" y el "respeto" en la escuela: formas culturales de participación en el marco de la disciplina escolar. En A. Furlán, C.L. Saucedo Ramos y B. Lara García (coords.), Miradas diversas sobre disciplina y violencia en centros educativos (pp. 35-51). Guadalajara, Jalisco: Universidad de Guadalajara.

Saucedo Ramos, C.L. (2005). Los alumnos de la tarde son los peores. Prácticas y discursos de posicionamiento de la identidad de alumnos problema en la escuela secundaria. Revista Mexicana de Investigación Educativa, 10(26), 641-668. Recuperado de https://www.redalyc.org/ articulo.oa?id=14002603

Spradley, J. P. (2016). Participant observation. Long Grove, IL: Waveland Press.

Tiramonti, G. (2004). La trama de la desigualdad educativa. Buenos Aires: Manantial.

Tomasini, M., Bertarelli, P. y Morales, M. G. (2017). Género, racialización de la clase e identidades: lasLas categorías "negros"'negros' y "negras"'negras' en jóvenes de sectores populares de Córdoba. Psicoperspectivas, 16(2), 9-19. Dol: https://doi.org/10.5027/psicoperspectivas-vol16-issue2-fulltext-954

Tomasini, M., López, C. J., Bertarelli, P. y García Bastán, G. (2010). La pelea entre jóvenes: búsqueda de reconocimiento social en un cruce de miradas. En ॥ Congreso Internacional de Investigación y Práctica Profesional en Psicología xVII Jornadas de Investigación Sexto Encuentro de Investigadores en Psicología del Mercosur. Facultad de Psicología, Universidad de Buenos Aires.

Valles, M. (1999). Técnicas cualitativas de investigación social. Reflexión metodológica y práctica profesional. Madrid: Síntesis.

Vasilachis, I. (coord.) (2007). Estrategias de investigación cualitativa. Buenos Aires: Gedisa.

Vásquez Bronfman, A. y Martínez, I. (1996). La socialización en la escuela. Una perspectiva etnográfica. Barcelona: Paidós.

Weinstein, C. (1981). Classroom Design as an external conditioning to learning. Educational tecnology, 21(8), 12-19. Recuperado de https:// www.learntechlib.org/p/164152

Weiss, E. (2009). Jóvenes y bachillerato en México: el proceso de subjetivación, el encuentro con los otros y la reflexividad. Propuesta Educativa, (32), 83-94. Recuperado de http://www.propuestaeducativa. flacso.org.ar/archivos/articulos/18.pdf 
Wenetz, I. y Stigger, M. (2011). Género y sexualidad en la escuela: un estudio etnográfico del recreo. En D. Milstein et al. (coords.), Encuentros etnográficos con niñ@s y adolescentes. Entre tiempos y espacios compartidos (pp. 51-76). Buenos Aires: Miño y Dávila.

Willis, P. (1988). Aprendiendo a trabajar. Cómo los chicos de la clase obrera consiguen trabajos de la clase obrera. Madrid: Akal.

Ziegler, S. (2016). Asimetrías entre profesores y estudiantes: disputas emocionales e ideológicas ante la formación de las élites. Revista Colombiana de Educación, (77), 101-124. Dol: https://doi.org/10.17227/01203916.70rce101.123 